\title{
Inertia Estimation of Spacecraft Based on Modified Law of Conservation of Angular Momentum
}

\author{
Dong Hoon $\mathrm{Kim}^{1 \dagger}$, Dae-Gyun $\mathrm{Choi}^{2}$, and Hwa-Suk $\mathrm{Oh}^{2}$ \\ ${ }^{1}$ Department of Aerospace Engineering, Texas A\&M University, H.R. Bright Building, Ross Street, College Station TX \\ 77843-3141, USA \\ ${ }^{2}$ School of Aerospace and Mechanical Engineering, Korea Aerospace University, Goyang 412-791, Korea
}

In general, the information of inertia properties is required to control a spacecraft. The inertia properties are changed by some activities such as consumption of propellant, deployment of solar panel, sloshing, etc. Extensive estimation methods have been investigated to obtain the precise inertia properties. The gyro-based attitude data including noise and bias needs to be compensated for improvement of attitude control accuracy. A modified estimation method based on the law of conservation of angular momentum is suggested to avoid inconvenience like filtering process for noiseeffect compensation. The conventional method is modified and beforehand estimated moment of inertia is applied to improve estimation efficiency of product of inertia. The performance of the suggested method has been verified for the case of STSAT-3, Korea Science Technology Satellite.

Keywords: inertia properties, angular momentum, moment of inertia, product of inertia, estimation of inertia matrix, STSAT-3

\section{INTRODUCTION}

In general, inertia properties of a spacecraft are measured before the launching and they are used in controlling the attitude for the spacecraft to perform its mission. However, the inertia properties are changed when situation that may affect the mass of the spacecraft occurs such as the decrease of the mass of the spacecraft by the consumption of propellant during the mission implementation. Moreover, when the mission requires detailed control of attitude, precise estimation of the inertia properties is necessary since change of them may have a great influence on the attitude control.

The attitude information of the spacecraft is used in estimating the inertia properties and the information includes noise. Since the inertia properties estimation by least squares method is susceptible to noise, noise-effect should be firstly reduced. To avoid such a procedure, an inertia properties estimation method based on the law of conservation of angular momentum was suggested and applied to actual estimation of inertia properties of a spacecraft (Peck 2000, Lee \& Wertz 2002). This method was studied for the cases where only one axis is maneuvered and where the moment of inertia is very large when compared to the product of inertia. One of drawbacks of this method is that the estimation error of the inertia is very large when all the three axes are maneuvered simultaneously. However, since it is impossible to maneuver only one axis when there exists product of inertia, the maneuver of three axes should be considered. In addition, accurate estimation of the product of inertia is necessary for the case of precise control.

In this paper, a modified method for inertia properties estimation based on the law of conservation of angular momentum is proposed for the case when three axes are maneuvered simultaneously. The process to reduce (c) This is an Open Access article distributed under the terms of the Creative Commons Attribution Non-Commercial License (http://creativecommons.org/licenses/by-nc/3.0/) which permits unrestricted non-commercial use, distribution, and reproduction in any medium, provided the original work is properly cited.
Received Jul 19, 2010 Revised Sep 20, 2010 Accepted Oct 01, 2010 †Corresponding Author

E-mail: donghoonkim@tamu.edu

Tel: +82-2-3159-0147 Fax: +82-2-300-0147 
the estimation error in the product of inertia is shown and the performance is verified by comparing the inertia properties estimation based on least squares method and the law of conservation of angular momentum as well as the proposed method.

\section{INERTIA PROPERTIES ESTIMATION TECHNIQUE}

\subsection{Inertia estimation based on least squares method}

The equation of motion including the control input by reaction wheels can be expressed as Eq. (1) (Hughes 1986):

$$
\begin{aligned}
& \dot{\omega}=I^{-1}\left[-\omega^{\times} I \omega-\omega^{\times} C I_{r w} \omega_{r w}+u\right] \\
& y=\omega
\end{aligned}
$$

where $I$ denotes the inertia matrix of the spacecraft, $\omega$ the angular velocity of the spacecraft, $I_{r v}$ the inertia matrix of the reaction wheel, $\omega_{r v}$ the angular velocity of the reaction wheel, $u$ the control input, $C$ the allocation matrix of the reaction wheel, and $y$ the measured angular velocity. Here, it is assumed that the three reaction wheels are exactly aligned with each axis.

For the inertia properties estimation based on least squares method, the equation is rearranged as Eq. (2):

$$
\mathrm{z}=H x
$$

where $H$ denotes the matrix constituted with the angular velocity of the spacecraft, $x$ the vector to be estimated, and $z$ the measured vector.

Using least squares method, the estimated value $\hat{x}$ can be obtained as in Eq. (3):

$$
\hat{x}=\left(H^{T} H\right)^{-1} H^{T} z
$$

Here, the elements of the matrix, $H$, are the information including noise. A rank of the matrix should be the maximum and the measured vector, $z$, should not be a zero vector (Simon 2006).

The variables in Eq. (3) are expressed as Eq. (4):

$$
\begin{aligned}
& \hat{x}=\left[\begin{array}{lllllllll}
\hat{I}_{x x} & \hat{I}_{x y} & \hat{I}_{x z} & \hat{I}_{y x} & \hat{I}_{y y} & \hat{I}_{y z} & \hat{I}_{z x} & \hat{I}_{z y} & \hat{I}_{z z}
\end{array}\right]^{T} \\
& H=\left[\begin{array}{ccccccccc}
\omega_{x} & \omega_{y} & \omega_{z} & 0 & 0 & 0 & 0 & 0 & 0 \\
0 & 0 & 0 & \omega_{x} & \omega_{y} & \omega_{z} & 0 & 0 & 0 \\
0 & 0 & 0 & 0 & 0 & 0 & \omega_{x} & \omega_{y} & \omega_{z}
\end{array}\right] \\
& z=\left[\begin{array}{lll}
Q_{x} & Q_{y} & Q_{z}
\end{array}\right]^{T}
\end{aligned}
$$

Here, the estimated value $\hat{x}$ represents the vector composed of moment of inertia and product of inertia. The elements of the measured vector $z, Q_{x}, Q_{y}$, and $Q_{z}$ are described in Section 2.2 .

\subsection{Inertia estimation based on the law of con- servation of angular momentum}

When a spacecraft is maneuvered using reaction wheels, all the angular momentum of the spacecraft is conserved in the inertial frame under the assumption that the disturbance is very small.

Defining $P$ as the matrix for the transformation from the inertial frame to the body frame, the angular momentum expressed in the inertial frame is as in Eq. (5) (Peck 2000, Lee \& Wertz 2002):

$$
H_{\text {inertial }}=P^{-1}\left[I \omega+C I_{r w} \omega_{r w}\right]
$$

Here, the transformation matrix $P$ is defined as Eq. (6), and each of the elements is constituted as quaternions.

$$
P=\left[\begin{array}{lll}
1-2\left(q_{2}^{2}+q_{3}^{2}\right) & 2\left(q_{1} q_{2}+q_{3} q_{4}\right) & 2\left(q_{1} q_{3}-q_{2} q_{4}\right) \\
2\left(q_{1} q_{2}-q_{3} q_{4}\right) & 1-2\left(q_{1}^{2}+q_{3}^{2}\right) & 2\left(q_{2} q_{3}+q_{1} q_{4}\right) \\
2\left(q_{1} q_{3}+q_{2} q_{4}\right) & 2\left(q_{2} q_{3}-q_{1} q_{4}\right) & 1-2\left(q_{1}^{2}+q_{2}^{2}\right)
\end{array}\right]
$$

According to the law of conservation of angular momentum, Eq. (7) is valid since the angular momentum should remain constant before and after the spacecraft maneuvering.

$P(t)^{-1}\left[I \omega(t)+C I_{r w} \omega_{r w}(t)\right] \approx P(0)^{-1}\left[I \omega(0)+C I_{r w} \omega_{r w}(0)\right]$

Assuming the initial angular velocity of the spacecraft to be 0 , the $I \omega(0)$ in Eq. (7) is set as 0 . In addition, assuming maneuvering of one axis of the spacecraft, the angular velocity of the other two axes is set as 0 . In the case of $\mathrm{x}$-axis maneuvering, the equation is reduced to Eq. (8a):

$$
\begin{aligned}
I\left[\begin{array}{c}
\omega_{x}(t) \\
0 \\
0
\end{array}\right] & =P(t) P(0)^{-1} C I_{r w} \omega_{r w}(0)-C I_{r w} \omega_{r w}(t) \\
Q(t) & =\left[\begin{array}{lll}
Q_{x} & Q_{y} & Q_{z}
\end{array}\right]^{T}
\end{aligned}
$$

Defining the right side of the Eq. (8a) as Eq. (8b) and inputting this in the Eq. (8a) again, Eq. (9) is derived by considering the first element:

$$
I_{x x} \omega_{x}(t)=Q_{x}(t)
$$

The estimated value $\hat{I}_{x x}$ can be obtained using least squares method as in Eq. (10):

$$
\hat{I}_{x x}=\left[\boldsymbol{\omega}_{\mathbf{x}}^{T} \boldsymbol{\omega}_{\mathbf{x}}\right]^{-1} \boldsymbol{\omega}_{\mathrm{x}}^{T} \mathbf{Q}_{\mathbf{x}}
$$

Here, $\boldsymbol{\omega}_{\mathrm{x}}$ and $\mathbf{Q}_{\mathrm{x}}$ are $\mathrm{N}$ by 1 column vectors. Repetition of 
the above method using $\left[\boldsymbol{\omega}_{x^{\prime}} \mathbf{Q}_{y}\right]\left[\omega_{x^{\prime}} \mathbf{Q}_{z}\right]$ gives the estimated values $\hat{I}_{y x}$ and $\hat{I}_{z \circ}$. Remaining elements can be estimated by the maneuvering of the $y$ - and $z$-axis. To obtain better estimated value, the estimated pair of product of inertia is averaged (Lee \& Wertz 2002).

\subsection{Inertia estimation based on modified law of conservation of angular momentum}

The inertia properties estimation method based on modified law of conservation of angular momentum is proposed to make it possible to estimate inertia properties in the case when three axes are maneuvered and to improve the accuracy of the product of inertia estimation.

Assuming that the angular momentum is constant before and after the spacecraft maneuvering, Eq. (7) can be arranged as follows:

$$
\left[\begin{array}{lll}
I_{x x} & I_{x y} & I_{x z} \\
I_{y x} & I_{y y} & I_{y z} \\
I_{z x} & I_{z y} & I_{z z}
\end{array}\right]\left[\begin{array}{l}
\omega_{x} \\
\omega_{y} \\
\omega_{z}
\end{array}\right]=\left[\begin{array}{l}
Q_{x} \\
Q_{y} \\
Q_{z}
\end{array}\right]
$$

Rewriting of the first row of Eq. (11) gives Eq. (12):

$$
I_{x x} \omega_{x}+I_{x y} \omega_{y}+I_{x z} \omega_{z}=Q_{x}
$$

Since the moment of inertia, $I_{x x}$, is relatively larger than the product of inertia, $I_{x y}$ and $I_{x z}$, in Eq. (12), the equation can be written as Eq. (13):

$$
I_{x x} \omega_{x} \approx Q_{x}
$$

By expressing the second and third row of Eq. (11) in the same manner shown above, the moment of inertia with respect to each axis can be estimated as in Eq. (14):

$$
\begin{aligned}
& \hat{I}_{x x}=\left[\boldsymbol{\omega}_{\mathrm{x}}^{T} \boldsymbol{\omega}_{\mathbf{x}}\right]^{-1} \boldsymbol{\omega}_{\mathrm{x}}^{T} \mathbf{Q}_{\mathbf{x}} \\
& \hat{I}_{y y}=\left[\boldsymbol{\omega}_{\mathrm{y}}^{T} \boldsymbol{\omega}_{\mathbf{y}}\right]^{-1} \boldsymbol{\omega}_{\mathrm{y}}^{T} \mathbf{Q}_{\mathbf{y}} \\
& \hat{I}_{z z}=\left[\boldsymbol{\omega}_{\mathrm{z}}^{T} \boldsymbol{\omega}_{\mathbf{z}}\right]^{-1} \boldsymbol{\omega}_{\mathrm{z}}^{T} \mathbf{Q}_{\mathbf{z}}
\end{aligned}
$$

The moment of inertia estimated by the procedure shown above is used for the calculation of the product of inertia as shown in Eq. (15):

$$
\begin{aligned}
& \tilde{I}_{x y}=\left[\boldsymbol{\omega}_{\mathbf{y}}^{T} \boldsymbol{\omega}_{\mathbf{y}}\right]^{-1} \boldsymbol{\omega}_{\mathbf{y}}^{T}\left[\mathbf{Q}_{\mathbf{x}}-\hat{I}_{x x} \boldsymbol{\omega}_{\mathbf{x}}\right] \\
& \tilde{I}_{x z}=\left[\boldsymbol{\omega}_{\mathbf{z}}^{T} \boldsymbol{\omega}_{\mathbf{z}}\right]^{-1} \boldsymbol{\omega}_{\mathbf{z}}^{T}\left[\mathbf{Q}_{\mathbf{x}}-\hat{I}_{x x} \boldsymbol{\omega}_{\mathbf{x}}\right] \\
& \tilde{I}_{y x}=\left[\boldsymbol{\omega}_{\mathbf{x}}^{T} \boldsymbol{\omega}_{\mathbf{x}}\right]^{-1} \boldsymbol{\omega}_{\mathbf{x}}^{T}\left[\mathbf{Q}_{\mathbf{y}}-\hat{I}_{y y} \boldsymbol{\omega}_{\mathbf{y}}\right] \\
& \tilde{I}_{y z}=\left[\boldsymbol{\omega}_{\mathbf{z}}^{T} \boldsymbol{\omega}_{\mathbf{z}}\right]^{-1} \boldsymbol{\omega}_{\mathbf{z}}^{T}\left[\mathbf{Q}_{\mathbf{y}}-\hat{I}_{y y} \boldsymbol{\omega}_{\mathbf{y}}\right] \\
& \tilde{I}_{z x}=\left[\boldsymbol{\omega}_{\mathbf{x}}^{T} \boldsymbol{\omega}_{\mathbf{x}}\right]^{-1} \boldsymbol{\omega}_{\mathbf{x}}^{T}\left[\mathbf{Q}_{\mathbf{z}}-\hat{I}_{z z} \boldsymbol{\omega}_{\mathbf{z}}\right] \\
& \tilde{I}_{z y}=\left[\boldsymbol{\omega}_{\mathbf{y}}^{T} \boldsymbol{\omega}_{\mathbf{y}}\right]^{-1} \boldsymbol{\omega}_{\mathbf{y}}^{T}\left[\mathbf{Q}_{\mathbf{z}}-\hat{I}_{z z} \boldsymbol{\omega}_{\mathbf{z}}\right]
\end{aligned}
$$

Eq. (15) indicates that the calculation for the estimation of the product of inertia is carried out using only the estimated moment of inertia. For example, in the procedure of calculating $\tilde{I}_{x z}$ in Eq. (15b), only the estimated $\tilde{I}_{x x}$ is used. However, considering Eq. (12), the $\tilde{I}_{x y}$ obtained in Eq. (15a) should be also considered. But, the moment of inertia estimated by Eq. (14) is a little larger than the actual value since the effect of product of inertia is not taken into account and, if both $\tilde{I}_{x x}$ and $\tilde{I}_{x y}$ that are obtained previously are used in estimating $\tilde{I}_{x z}$, the error of $\tilde{I}_{x z}$ will be increased more. Hence, only the moment of inertia is used, not the product of inertia obtained previously.

The pair of product of inertia estimated in the same conventional method is averaged for the calculation as follows:

$$
\begin{aligned}
& \hat{I}_{x y}=\hat{I}_{y x}=\frac{1}{2}\left[\tilde{I}_{x y}+\tilde{I}_{y x}\right] \\
& \hat{I}_{x z}=\hat{I}_{z x}=\frac{1}{2}\left[\tilde{I}_{x z}+\tilde{I}_{z x}\right] \\
& \hat{I}_{y z}=\hat{I}_{z y}=\frac{1}{2}\left[\tilde{I}_{y z}+\tilde{I}_{z y}\right]
\end{aligned}
$$

\begin{tabular}{|c|c|c|c|}
\hline \multicolumn{2}{|c|}{ Items (units) } & \multirow{2}{*}{\multicolumn{2}{|c|}{$\begin{array}{c}\text { Values } \\
10^{-4}\end{array}$}} \\
\hline Noise level & $\sigma_{\mathrm{s}}(\mathrm{rad} / \mathrm{s})$ & & \\
\hline Spacecraft inertia & $I\left(\mathrm{~kg} \cdot \mathrm{m}^{2}\right)$ & {$\left[\begin{array}{l}13.916 \\
0.0850 \\
0.1330\end{array}\right.$} & $\left.\begin{array}{ll}0.0850 & 0.1330 \\
16.954 & 0.5896 \\
0.5896 & 19.894\end{array}\right]$ \\
\hline Wheel inertia & $I_{r w}\left(\mathrm{~kg} \cdot \mathrm{m}^{2}\right)$ & \multicolumn{2}{|c|}{$4.113 \times 10^{-4} \times \operatorname{diag}\left\{\begin{array}{lll}1 & 1 & 1\end{array}\right\}$} \\
\hline Natural frequency & $\omega_{n}(\mathrm{rad} / \mathrm{s})$ & \multicolumn{2}{|c|}{0.043} \\
\hline Damping ratio & $\zeta$ & \multicolumn{2}{|r|}{0.707} \\
\hline Control gains & $\begin{array}{l}K_{P} \\
K_{D}\end{array}$ & \multicolumn{2}{|r|}{$\begin{array}{c}2 \omega_{n}^{2} I \\
2 \zeta \omega_{n} I\end{array}$} \\
\hline Initial states & $\begin{array}{c}\theta_{i}(\mathrm{deg}) \\
\omega_{i}(\mathrm{rad} / \mathrm{s}) \\
\omega_{r w}(\operatorname{rad} / \mathrm{s})\end{array}$ & \multicolumn{2}{|c|}{$\begin{array}{l}{\left[\begin{array}{lll}10 & 10 & 10\end{array}\right]^{T}} \\
{\left[\begin{array}{lll}0 & 0 & 0\end{array}\right]^{T}} \\
{\left[\begin{array}{lll}0 & 0 & 0\end{array}\right]^{T}}\end{array}$} \\
\hline Target states & $\begin{array}{c}\theta_{t}(\mathrm{deg}) \\
\omega_{t}(\mathrm{rad} / \mathrm{s})\end{array}$ & \multicolumn{2}{|r|}{$\begin{array}{l}{\left[\begin{array}{lll}0 & 0 & 0\end{array}\right]^{T}} \\
{\left[\begin{array}{lll}0 & 0 & 0\end{array}\right]^{T}}\end{array}$} \\
\hline Wheel allocation & C & \multicolumn{2}{|c|}{$\operatorname{diag}\left\{\begin{array}{lll}1 & 1 & 1\end{array}\right\}$} \\
\hline
\end{tabular}

\section{SIMULATION RESULTS AND ANALYSIS}

For the comparison of the inertia properties estimation methods mentioned above, a numerical simulation is performed. The simulation parameters are listed in Table 1.

The controller in Eq. (17) is used for the attitude control.

$$
u=-K_{P} q_{e}-K_{D} \omega_{e}
$$

Table 1. Simulation parameters. 
where $u$ denotes the control input, $q_{e}$ the quaternion error, $\omega_{e}$ the angular velocity error, $K_{p}$ the proportional gain, and $K_{D}$ the derivative gain.

The angle, the angular velocity of the spacecraft, and the control input from the numerical simulation are shown in Figs. 1 and 2. Based on these data, the inertia properties are estimated by the methods mentioned above and the results are shown in Tables 2 and 3, and Figs. 3 and 4, respectively.

In Table 2, "True" indicates the actual inertia properties of the spacecraft, "Batch" the values estimated by using least squares method, "Conventional" the values estimated by using the conventional method based on the law of conservation of angular momentum, "Considering pre-estimate" the estimated values obtained by using the previously calculated product of inertia in the calculation of the next product of inertia, and "Suggested" the values estimated by using the method that the author propose.
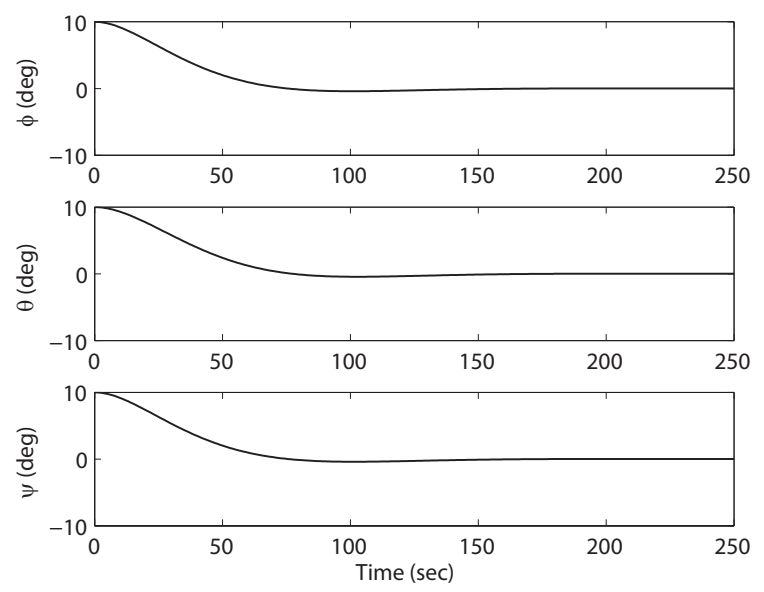

Fig. 1. Time trajectory of the Euler angles.

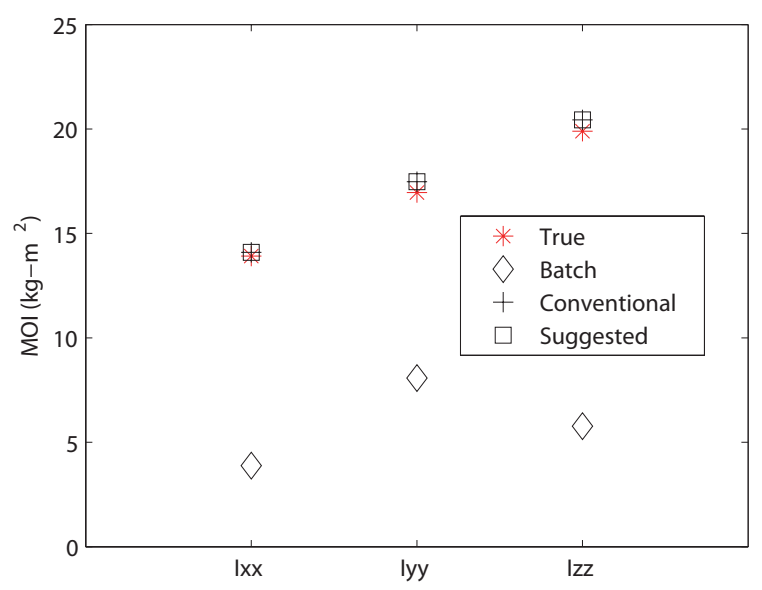

Fig. 3. Estimated moment of inertia.
Table 2. Estimation results $\left(\mathrm{kg} \cdot \mathrm{m}^{2}\right)$.

\begin{tabular}{lrrccc}
\hline & True & Batch & Conventional & $\begin{array}{c}\text { Considering } \\
\text { pre-estimate }\end{array}$ & Suggested \\
\hline$I_{x x}$ & 13.9160 & 4.2859 & 14.1181 & 14.1181 & 14.1181 \\
$I_{y y}$ & 16.9540 & 7.1157 & 17.5350 & 17.5350 & 17.5350 \\
$I_{z z}$ & 19.8940 & 6.1224 & 20.6816 & 20.6816 & 20.6816 \\
$I_{x y}$ & 0.0850 & 4.8224 & 16.3578 & -0.0019 & 0.1197 \\
$I_{y z}$ & 0.5896 & 6.1667 & 19.1201 & 0.0062 & 0.1538 \\
$I_{z x}$ & 0.1330 & 6.2786 & 17.4000 & 0.0589 & 0.1560 \\
\hline
\end{tabular}

Table 3. Comparison of the errors (\%).

\begin{tabular}{rrrcc}
\hline & \multicolumn{1}{c}{ Batch } & Conventional & $\begin{array}{c}\text { Considering } \\
\text { pre-estimate }\end{array}$ & Suggested \\
\hline$I_{x x}$ & 69.2018 & 1.4520 & 1.4520 & 1.4520 \\
$I_{y y}$ & 58.0296 & 3.4269 & 3.4269 & 3.4269 \\
$I_{z z}$ & 69.2248 & 3.9592 & 3.9592 & 3.9592 \\
$I_{x y}$ & $5,573.3560$ & $19,144.5041$ & 102.2033 & 40.8686 \\
$I_{y z}$ & 945.9131 & $3,142.9016$ & 98.9418 & 73.9096 \\
$I_{z x}$ & $4,620.7823$ & $12,982.6901$ & 55.6939 & 17.2956 \\
\hline
\end{tabular}
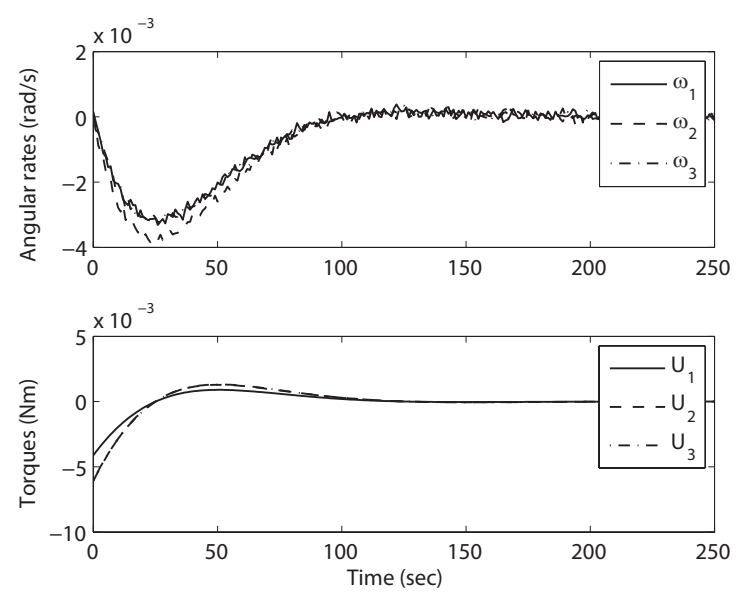

Fig. 2. Time trajectory of the angular rates and the control inputs.

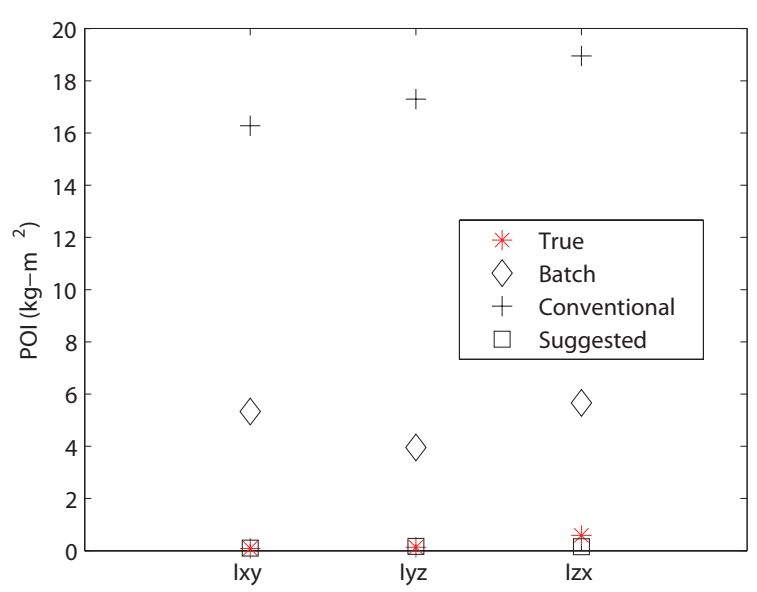

Fig. 4. Estimated product of inertia. 
Quite large errors are found between the estimated values and the actual inertia properties in the case where least squares method is used. When this method is applied, nine inertia properties are calculated at once as shown in Eqs. (3) and (4). However, as described in Section 2.3 , least squares method is individually applied for the calculation of the nine individual inertia properties when the law of conservation of angular momentum is used. For example, in the process of calculating $\hat{I}_{x x}$, the effect of not only the $x$-axis angular velocity but also the $y$ - and z-axis angular velocities are included when using least squares method. On the contrary, when the law of conservation of angular momentum is used, only the $\mathrm{x}$ axis angular velocity is involved in the calculation of $\hat{I}_{x x}$. As such, the error is larger when least squares method is used because of the effect of the information of the axis related with the inertia properties to be estimated as well as the effect of the information of the other axes. Moreover, since this method is sensitive to noise, the procedure to compensate noise-effect with a filter is necessary for accurate estimation.

In the case when the conventional method based on the law of conservation of angular momentum is used, the moment of inertia is well estimated, but the error is very large in the product of inertia. This is because, when all the three axes are maneuvered, the maneuvering of the other two axes is not considered.

The proposed method shows the same error in the estimated moment of inertia with that of the conventional method based on the law of conservation of angular momentum. However, relatively better results are obtained in the product of inertia when compared with the two methods mentioned previously. As noted in Section 2.3, in the calculation of product of inertia, less error is found when the product of inertia calculated in advance is not used in the estimation of the product of inertia. In addition, among the product of inertia estimated by the proposed method, $\hat{l}_{y z}$ shows a large error. This is because $\hat{I}_{y y}$ and $\hat{I}_{z z}$ of which errors are larger than that of $\hat{I}_{x x}$ are used for the estimation of $\hat{I}_{y z}$. Even though the absolute magnitude of the error is large, the error can be considered not very large from the view of the overall estimated values, because the magnitude of the product of inertia itself is relatively very small.

\section{CONCLUSIONS}

The study in this paper is conducted by focusing on the following three aspects: first, simultaneous maneuvering of three axes is considered since maneuvering of only one axis is not possible where there exists product of inertia; second, when using the attitude information including noise, the conventional attitude information is used without the noise-effect compensation procedure using filters; finally, an accurate method for the estimation of product of inertia is proposed, which showed far better result than that of the conventional methods.

In this study, inertia properties are estimated by one time of calculation. In the future study, convergence of the estimated values will be verified by using iteration technique.

\section{ACKNOWLEDGEMENTS}

This work supported by Research Program supported by the Ministry of Education, Science and Technology (STSAT-3 program), Korea. Authors fully appreciate the financial support.

\section{REFERENCES}

Hughes, P. C. 1986, Spacecraft Attitude Dynamics (New Jersey: John Wiley \& Sons, Inc.)

Lee, A.Y. \&Wertz, J. A. 2002, JSpRo, 39, 153, doi: 10.2514/2.3795 Peck, M. A. 2000, AdAeS, 103, 2005

Simon, D. 2006, Optimal State Estimation: Kalman, H-infinity, and Nonlinear Approaches (Hoboken: Wiley-Interscience) 